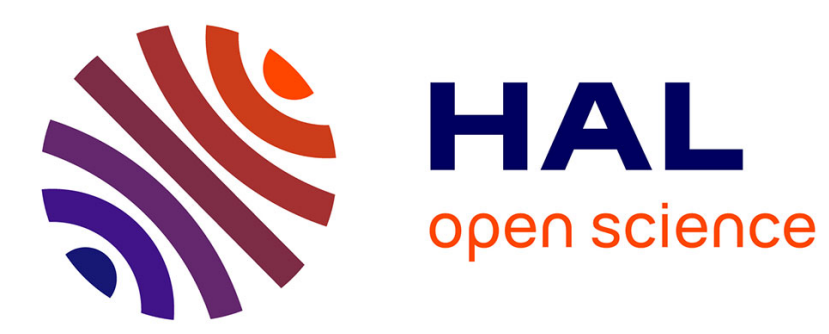

\title{
Rencontre avec un braconnier de l'archive
}

Noël Barbe, Jean-Christophe Sevin

\section{To cite this version:}

Noël Barbe, Jean-Christophe Sevin. Rencontre avec un braconnier de l'archive. Sociétés \& Représentations, 2005, Lieux d'archive, 19, pp.65-75. 10.3917/sr.019.0065 . halshs-01933348

\section{HAL Id: halshs-01933348 \\ https://shs.hal.science/halshs-01933348}

Submitted on 23 Nov 2018

HAL is a multi-disciplinary open access archive for the deposit and dissemination of scientific research documents, whether they are published or not. The documents may come from teaching and research institutions in France or abroad, or from public or private research centers.
L'archive ouverte pluridisciplinaire HAL, est destinée au dépôt et à la diffusion de documents scientifiques de niveau recherche, publiés ou non, émanant des établissements d'enseignement et de recherche français ou étrangers, des laboratoires publics ou privés. 


\section{RENCONTRE AVEC UN BRACONNIER DE L'ARCHIVE \\ Noël Barbe, Jean-Christophe Sevin}

Éditions de la Sorbonne | "Sociétés \& Représentations 》

2005/1 N 19 | pages 65 à 75

ISSN 1262-2966

Article disponible en ligne à l'adresse :

https://www.cairn.info/revue-societes-et-representations-2005-1-page-65.htm

\section{Pour citer cet article :}

Noël Barbe, Jean-Christophe Sevin « Rencontre avec un braconnier de l'archive », Sociétés \& Représentations 2005/1 ( $\left.{ }^{\circ} 19\right)$, p. 65-75.

DOI 10.3917/sr.019.0065

Distribution électronique Cairn.info pour Éditions de la Sorbonne.

(c) Éditions de la Sorbonne. Tous droits réservés pour tous pays.

La reproduction ou représentation de cet article, notamment par photocopie, n'est autorisée que dans les limites des conditions générales d'utilisation du site ou, le cas échéant, des conditions générales de la licence souscrite par votre établissement. Toute autre reproduction ou représentation, en tout ou partie, sous quelque forme et de quelque manière que ce soit, est interdite sauf accord préalable et écrit de l'éditeur, en dehors des cas prévus par la législation en vigueur en France. Il est précisé que son stockage dans une base de données est également interdit. 


\section{RENCONTRE AVEC UN BRACONNIER DE L'ARCHIVE}

\section{Noël Barbe et Jean-Christophe Sevin}

"Sélectionner les cas singuliers et les scènes mineures est plus important que toute considération d'ensemble1."

C'est en 2003 que nous avons rencontré, à plusieurs reprises, M. Rebugai. Nous menions alors un travail d'enquête dans le cadre d'une exposition prévue au musée d'archéologie du Jura, à Lons-le-Saunier: «Patrimoines singuliers 2 ». Loin des pratiques habituelles de l'archéologie qui convoque rarement des hommes préhistoriques autour d'une table de discussion, ou même des musées dits de société3 qui réduisent le plus souvent la parole de l'autre à une fonction documentaire, ce musée entendait ouvrir son espace d'exposition, le temps d'un été, en demandant à ceux qui le voulaient de prêter un objet qu'ils qualifiaient de patrimonial.

À la question commune posée par le musée d'archéologie: «Qu'est-ce qui, pour vous, évoque le patrimoine et la mémoire?», la réponse est, pour M. Rebugai, évidente, même si elle s'avère pour nous étonnante: soixante tonnes de papier.

M. Rebugai «fait les déchetteries» pour, du papier quil y trouve, faire «avec ses bras», des papiers. Il y rencontre parfois ceux qui s'en débarrassent, plus rarement il est appelé

1. Gilles Deleuze, Critique et Clinique, Paris, Minuit, 1993, p. 77.

2. Cette exposition s'est accompagnée du tournage d'un film documentaire, Votre patrimoine nous intéresse, réalisé par Jean-Luc Bouvret. Les résultats de l'enquête ont été partiellement publiés. Cf. Noël Barbe, Émilie Denêtre et Jean-Christophe Sevin, "Une archéologie des patrimoines", journal de l'exposition Patrimoines singuliers, Musée d'archéologie de Lons-le-Saunier, 2003, pp. 17-20.

3. Le terme est employé par la direction des musées de France, depuis un colloque de 1991, pour désigner des musées qui ont pour objectif d'étudier l'évolution de l'humanité dans ses composantes sociales et historiques et de faire comprendre la diversité des cultures et des sociétés. 
pour vider un grenier. Rien à voir ici avec une vocation historienne, une volonté de conserver les traces de la grandeur de l'État-Nation ou d'une aventure industrielle. Plutôt une sorte de destin commun qui se noue là, dans une décharge, entre «un document simple» rejeté bien qu' «intéressant» et un homme souhaitant se nourrir lui et les siens alors qu'il est lui-même exclu d'un monde, celui du travail. Comme maintenant encore lorsquili descend chercher réconfort dans la cave où il travaille sur ses papiers quand le moral vient à lui manquer. Une sorte d'adoption de ces choses abandonnées, une parenté tissée, au risque de l'incompréhension, de l'assimilation du collecteur à la nature de la récolte, d'une incapacité à distinguer l'un de l'autre. Une histoire personnelle donc, placée sous le signe d'un événement porteur de possiblex : s’il n'est pas seul «à faire les poubelles», tous ceux qui le font ne se transforment pas en archiviste.

\section{"Je traversais une période très difficile»}

"J'ai commencé la collecte en petite quantité il y a une vingtaine d'années et depuis douze ans j'ai créé une association pour la sauvegarde du patrimoine écrit. Maintenant c'est par centaines de kilos que je rentre les documents. Je traversais une période très difficile, j'étais au chômage et puis j'étais allé voir simplement les décharges pour trouver des choses à vendre et donner à manger à ma famille et j’ai découvert ce fameux bout de papier, peut-être un coup de chance. C'était un petit courrier, un document simple mais intéressant. Il m’a donné le déclic pour aller en chercher un deuxième puis un dixième puis un millième et d'en être où j'en suis. J'ai à peu près soixante tonnes de documents, pour vous donner une idée.

Personne ne s'y intéressait à l'époque. C'était des cochonneries, c'était des vieux papiers, des vieux journaux. Bon, vulgairement, c'était de la m..., personne ne les regardait. Et puis je me suis assis un jour au bord des trucs et j’ai commencé à lire. Je me suis dit: "tiens, à tel endroit il y a eu la fête, il y a eu tel fait, c'est assez intéressant". Après, on trouve quelque chose un petit peu plus historique, un petit peu plus intéressant et on met le doigt dedans et on est foutu (rire). Mais je considère ça comme une bonne maladie. Avant, $j$ habitais la Marjorie5. Quand je rentrais avec mes cartons de papiers on disait que j'étais pas bien. Les gens ne comprenaient pas pourquoi je récupérais tout ce tas de saloperies sans intérêt.

Je fais les déchetteries, avant c'était les poubelles, mot un peu plus vulgaire, maintenant c'est les déchetteries. Avant j'allais dans les bennes après que les gars sont passés, je restais de sept heures du soir à cinq heures du matin pour fouiller. Je descendais dans la benne, je fouillais, je brassais les kilos de papier. C'est long. Maintenant c'est du papier

4. François Zourabichvili, "Deleuze et le possible», in Gilles Deleuze, une vie philosophique, Paris, Les Empêcheurs de penser en rond, 1998, pp. 335-357.

5. Il s'agit d'un quartier de Lons-le-Saunier.

N. Barbe, J.-C. Sevin, "Rencontre avec un braconnier...", S. \& R., n 19, Avril 2005, pp. 65-75. 
ça va plus vite. Dans le temps il y avait de tout, alors il fallait trier parmi ce tas de cochonneries. J'arrive toujours à sortir un ou plusieurs petits documents. Là-dedans on retrouve la mémoire des gens de toute une époque, des siècles passés. Il y a très, très longtemps comme un petit peu moins longtemps. C'est la vie de tous les jours.

Ma plus grosse récupération a duré une nuit complète. J'ai fait sept voyages avec mon véhicule, sept aller-retour entre la déchetterie et la maison. C'était un commerce sur Lons qui se vidait, donc là j’ai récupéré des grosses quantités.

Quelquefois quand les gens sont à la déchetterie et que je suis là au bon moment, ils me demandent si ça m’intéresse et je récupère directement une grosse quantité de papier, au lieu d'aller fouiller dans la benne.

Là dans les semaines qui viennent, je vais prendre mes bras et je vais aller chercher à peu près cent cinquante kilos de journaux. C'est très rare que l'on m'appelle, mais c'est ce que je souhaiterais. Ce serait mieux pour la conservation, ça serait moins éparpillé. Parce que quand on jette, on mélange tout. Donc si c'est un document intéressant, il faut tout reclasser. C'est vrai que mon souhait serait que les gens me contactent. Ça arrive mais malheureusement très, très peu parce que quand la personne meurt ce sont les enfants ou des gens qui n'ont aucun intérêt avec la famille qui récupèrent la maison. Il faut donc faire très vite parce que le temps est là pour pousser et on pense pas que des papiers qui encombrent le grenier intéressent quelqu'un. Ce n'est pas la même démarche que pour un antiquaire où l'on sait qu'un meuble peut valoir de l'argent. Il n'y a pas forcément le rapport argent.»

\section{"Il n’y a pas d'argent»}

Le fonctionnement de l'association qu'il a créée et la circulation des papiers entre les hommes reposent sur le refus d'un formatage par l'argent qui rendrait obligatoires les écritures comptables et corromprait les relations entre les êtres. Point donc d'outil de gouvernement des hommes et des choses qui introduirait un principe de mise en équivalence. Ces choses-là ne sont pas "marchandisables» pas plus que les liens entre les hommes qui entrent en relation à leur propos. La singularité de chaque papier est posée, seul donc le principe de similitude peut introduire à leur échange. L'intérêt premier est celui des papiers qu'il faut, avec une prévalence du plaisir, trouver, classer, mettre en ordre et en valeur; leur circulation par ailleurs réduite pouvant permettre l'instauration d'un lien social durable.

«Il n'y pas de cotisation pour l'association. Nous sommes une dizaine, les membres de l'association viennent surtout pour classer et mettre en ordre quand nous faisons des expositions. Le but c'est la motivation, c'est d'avoir envie de fouiller et de mettre en valeur ces bouts de papier qui ne ressemblent à rien mais qui ont un intérêt quand même. C'est sans but lucratif, c'est simplement le plaisir de fouiller le papier, de le découvrir, de 
lire le contenu et puis de faire des expositions pour montrer aux gens. Et peut-être les impliquer aussi en leur disant d'arrêter de jeter leurs papiers qui leur semblent bons à rien, ils ont peut-être un intérêt.

Je ne veux pas me servir de l'association pour faire de l'argent, c'est un loisir. Le pêcheur, quand il va au bord de l'eau et qu'il pêche, s'il ne prend pas de poisson, n'envoie pas de facture. S'il a cassé sa ligne, il n'envoie pas à la société de pêche la ligne que le poisson a cassée. Donc, pour moi, le poisson c'est le papier. Mon plaisir c'est ça et il n'y a pas d'argent.

S'il y a deux exemplaires d'un document et que, à la rigueur, ça fait plaisir à quelqu'un, je lui donne. Je ne concevrais pas non plus de le vendre parce que je ne l'ai pas payé. Bien sûr ça alimenterait l'association, ça ferait une petite rentrée d'argent, mais est-ce que ça vaut le coup, dans une passion, de faire rentrer le côté argent? Parce que je m'en suis rendu compte sur d'autres activités, bien souvent, dès qu'on parle argent on fout en l'air le système. Et puis, est-ce que pour rentrer dix francs dans l'année, il faut se prendre la tête à faire tout un fichier de comptabilité? Alors que le gars il me donne une feuille de cahier, moi je lui donne un prospectus et on est tous les deux contents. On n'a pas d'argent donc on n'a pas de mauvais rapports entre nous. Et puis je pense que c'est beaucoup plus simple, enfin ça fait douze ans que je tourne comme ça. Mais des propositions je peux vous dire que j'en ai eues, les gars quand ils viennent: "oui je te l'achète". Parce que c'est pareil, l'argent c'est aussi le côté où on vous prend, on vous donne et puis il y a rupture. Vous ne devez rien, c'est ça contre ça, vous rentrez chez vous et on ne se connait plus. Il est vrai que quand vous donnez quelque chose, si ça fait plaisir, aussi bien d'un côté que de l'autre, vous êtes redevable. Il y a un lien qui nous tient, parce qu'il m'a donné un truc qui me plaisait et que je ne lui ai pas rendu tout de suite, ou je lui ai rendu autre chose. Il y a ce côté-là aussi qui crée peut-être quelque chose de plus fort qu'un billet de cent francs.

Moi, c'est à but non lucratif. C'est pour ça que je vous disais tout à l'heure que j’ai eu énormément de difficultés avec des gens, parce quilis avaient tendance à dire: "ça vaut tant, il tourne le dos, on lui pique et on y vend ". Mais je ne suis pas bête, je connais aussi les prix de tel ou tel document. Je sais le faire mais si je ne le fais pas c'est parce que c'est pas l'objectif que je me suis fixé.»

\section{"Le papier c'est la trace de la mémoire»}

Les papiers recueillis par M. Rebugai sont hétérogènes. On ne peut les assigner à des catégories comme les archives familiales, les archives d'institutions, les archives liées à l'élaboration d'une œuvre artistique ou scientifique. On pourrait à la limite les assimiler à des archives individuelles dans le sens où elles sont produites par les activités de M. Rebugai. Au-delà, ce qui les rassemble, c'est leur caractère de support de l'écriture qu'elle soit manuscrite, imprimée ou figurative, leur caractère de support de l'inscription

N. Barbe, J.-C. Sevin, «Rencontre avec un braconnier... », S. \& R., nº 19, Avril 2005, pp. 65-75. 
"de l'importance des choses vécues». C'est dans ces choses vécues que plonge M. Rebugai, vivant raconte-t-il plusieurs vies 6 . Le papier pour l'écriture... Des personnes en choses...7

" Ça peut être une lettre d'une personne à une autre, quelle que soit la personne, pendant une période bien particulière comme la guerre. Des documents qui sont en général la petite histoire de la région. Mais c'est aussi bien un Paris-Match qu'un document intéressant, des journaux, des livres, des cartes postales, des photos, des dépliants publicitaires, une revue d'une association quelconque, quelque chose qui retrace un passage de la vie d'une époque, c'est en général du temps passé.

Le papier c'est la trace de la mémoire, sur lequel on raconte une belle journée. Ça peut aussi être important. Ça peut être un document administratif. On laisse sur papier l'importance des choses vécues. C'est pour ça que je me suis intéressé au support papier qui laisse des traces et qui est une mémoire.

Je trie, et quand je suis là je suis ailleurs, je suis dans un autre univers, ça me change l'esprit. J'ai l'impression de vivre deux ou trois vies. Parce que quand vous replongez cinquante, cent ou cent cinquante ans en arrière, vous essayez de resituer un peu le courrier ou le document et vous finissez par connaître un petit peu la personne, "oui il faisait ça, il y avait ça qui était avec", il vous arrive même de mélanger un petit peu l'ancien et le futur. C'est-à-dire vous trouvez un courrier. Ou vous le prenez bêtement et vous écrivez "courrier 1870" et vous le mettez dans une case. Ou alors vous vous demandez ce quil dit, vous le nettoyez un petit peu, vous le dépoussiérez, vous commencez à lire l'en-tête, vous allez un petit peu plus loin parce que vous trouvez ça sympa. Puis il y aura un moment, il y aura quelque chose qui manque ou que vous ne comprenez pas parce que c'est une autre façon de vivire. Le but c'est de chercher, de prendre des renseignements. "

\section{"... je les trie»}

Comme tout archiviste, M. Rebugai redistribue dans l'espace8. Les papiers passent de la déchetterie à la cuisine, puis dans une des pièces de la cave - "le garde-manger» avant d'intégrer des cartons - en un classement opératoire qui vise tout à la fois à

\footnotetext{
6. Mais ceci n'est-il pas vrai aussi des chercheurs en sciences sociales? Décrivant le travail de réécoute de ses entretiens enregistrés, Jean-Claude Kaufmann écrit: "Je me laisse entraîner, par l'histoire dans laquelle j'entre progressivement, par les hypothèses en mouvement dans ma tête... " (L'Entretien compréhensif, Paris, Nathan, 1996, p. 80). Paul Ricœur souligne également «la dimension d'intersubjectivité de la connaissance historique» (La Mémoire, l'bistoire, l'oubli, Paris, Le Seuil, 2000, p. 437).

7. Il s'agit là d'un clin d'œil à Laurent Thévenot ("Le régime de familiarité. Des choses en personne», Genèses, $n^{\circ} 17,1994$, pp. 72-101).

8. Michel de Certeau, L'Écriture de l'histoire, Paris, Gallimard, 1975, p. 84.
}

N. Barbe, J.-C. Sevin, «Rencontre avec un braconnier... ", S. \& R., n 19, Avril 2005, pp. 65-75. 
préserver et à construire leur caractère documentaire - et de migrer en d'autres lieux de stockage. Dans les services départementaux d'archives, l'espace et la répartition des "documents" sont communs dans le sens où les principes qui les gouvernent ne dépendent pas de l'identité propre de l'agent qui les manipule. Ici au contraire nous avons affaire à un homme avec son propre corps et sa topographie particulière, tout à la fois informée et informant la distribution des papiers. C'est ce corps à nourrir qui les lui a fait rencontrer, c'est lui qui, en dehors de toute cartographie extrinsèque, devient le "classeur» des fonds constitués. C'est lui le cartographe d'un espace habité, là où on ne rencontre généralement qu'une cartographie inscrite de traces écrites. Ce besoin de lui pour ne pas se perdre en mer$^{9}$ provoque un curieux renversement des rapports entre l'oralité et l'écriture. L'archive naît selon Paul Ricœur du basculement du champ de l'oralité dans celui de l'écriture ${ }^{10}$. Ici point d'archive sans oralité.

"Quand je rentre juste avant de manger, que j’ai fait ma journée de travail, que j’ai pris mon petit quart d'heure, ma demi-heure pour aller fouiller, et que je pose le carton de vieux papiers, il y a la poussière et il y a l'odeur. Quand je n'ai pas eu le temps de les trier, ils restent à la maison la journée. Le lendemain, pendant la nuit, quand tout le monde est couché, j’ai un peu de place à la maison alors je les trie.

J'essaie de faire des thèmes quand c'est possible. Si c'est un dossier c'est peut-être pas conseillé de le défaire. Je n’ai pas de formation. Qu'est-ce que vous faites quand c'est des dossiers? Est-ce que vous les démontez en disant c'est économique, c'est politique, c'est architectural, c'est la vie de tous les jours parce qu'il y a des factures? Ce n'est pas facile. Alors c'est un choix que je me suis donné pour classer, répertorier. J'essaye aussi de ne pas trop mélanger. Si on mélange trop, après on ne comprend plus pourquoi telle ou telle photo. Si c'est un paquet de photos, il faut laisser le paquet de photos. Il n'y a déjà pas de nom sur ces photos, si après on les mélange encore avec d'autres, disons qu'on détruit le document par lui-même. Donc il faut aussi laisser un ensemble de documents, j'essaie de ne pas trop défaire les dossiers. Si c'est un livre c'est vite fait, c'est de l'éducation ou c'est de l'histoire, de la géographie. Le livre ne pose pas de problème, le papier est beaucoup plus difficile à classer.

Pour ce qui est du rangement, au début je prenais des cartons un peu de toutes formes, je stockais ça n'importe comment. Et je me suis rendu compte au bout d'un moment que le carton se tasse et que les piles tombent. Maintenant j'ai un petit peu standardisé, je récupère certains types de cartons, j'ai normalisé pour que les piles se tiennent, vous voyez. Disons que c'est l'école de la vie. On ne peut pas le savoir, c'est en apprenant qu'on devient meilleur, il n'y a pas de formation, il n'y a pas d'école.

9. Pour paraphraser Arlette Farge (Le Goût de l'archive, Paris, Le Seuil, 1989, p. 11).

10. Paul Ricoeur, La Mémoire, l'histoire, l'oubli, op. cit., pp. 181-182.

N. Barbe, J.-C. Sevin, "Rencontre avec un braconnier...", S. \& R., nº 19, Avril 2005, pp. 65-75. 
La première démarche c'est de sauver et de conserver. Après c'est ma mémoire qui fait un petit peu classeur. Connaissant un peu quand même tout ce que j'ai, quand on a besoin je prends là ou là, selon ce que je prépare comme truc. Quand j’ai besoin pour une exposition, je vais chercher telle chose sans fiche, sans ordinateur, par question de temps, question d'argent. Il faut que j'aie un peu de temps pour les dépoussiérer, les remettre un petit peu en ordre, parce que souvent c'est tout un tas de papiers dans tous les sens."

\section{"...mis à disposition..."}

Le devenir des papiers recueillis est double. Tout d'abord ils sont fractionnés - première des redistributions spatiales - au nom d'un partage, sociologiquement armé, entre "grande» et "petite» histoire. Les documents relevant de la première sont «rendus" à l'espace dont ils sont accidentellement sortis, tandis que les seconds - ceux qui sont vraiment abandonnés - rejoignent les réserves de $\mathrm{M}$. Rebugai.

Pour autant ils ne sont pas soustraits au monde, qưils soient exposés à la demande ou communiqués à des lecteurs intéressés. Il faut une nouvelle vie pour ces documents, que leurs usages soient tournés vers la connaissance ou l'action, sans quoi leur sauvetage serait marqué du sceau de la vacuité.

"Il y a l'histoire que l'on connaît, c'est-à-dire celle des rois, celle des célébrités. Et il y a celle des gens dont on parle beaucoup moins et je pense que c'est intéressant d'en garder l'histoire et le patrimoine, c'est pour ça aussi que j'ai créé cette association.

Les poubelles ce n'est pas forcément que les cochonneries non plus, il y a aussi notre histoire qui peut se glisser dedans. Alors il y a la grande histoire et l'histoire des gens plus simples, comme moi, de tous les jours. Et il est vrai que quand je trouve un document important, je le remets là où il n'aurait jamais dû s'en aller. Bien souvent c'est des documents historiques ou administratifs donc ils auraient dû rester dans les administrations et avec le temps, les guerres, enfin des situations bien particulières ils ont bougé. Moi jes-" saie quand même de rester sur l'histoire de monsieur tout le monde. Une journée, une façon de voir le paysage du Jura par exemple. Les documents les plus intéressants, je les verse aux Archives départementales parce que c'est des choses que je ne peux pas conserver mais que l'on peut utiliser. J'ai eu la chance de récupérer une charte de franchise de 1341, c'est un document beaucoup plus historique, si je ne l'avais pas récupéré dans la décharge, il était détruit à tout jamais.

J'ai aussi donné énormément de documents au musée militaire, parce que les gens ne pensent pas que l'armée peut avoir un musée et s'intéresser à son histoire, qui est aussi la nôtre. J'ai donné au 44e régiment d'infanterie à Lons des plans des casernes de Nancy. Le

N. Barbe, J.-C. Sevin, «Rencontre avec un braconnier... », S. \& R., n 19, Avril 2005, pp. 65-75. 
maitre d'œuvre était un lédonien ${ }^{11}$, mais comme c'était Nancy, c'est vrai que je me sentais un peu moins concerné et comme il y avait une structure qui était là pour accueillir ces documents c'était aussi bien quilils soient conservés dans la structure qui va les utiliser plutôt que dans mes sous-sols où j’ai déjà une grosse quantité de papier.

Ce n'est pas par égoïsme que je vais garder ces documents en me disant que je les ai et qu'eux ne les ont pas. Il faut que ces documents revivent une nouvelle fois, il faut qu'il y ait une suite. Si c'est pour les laisser pourrir dans un coin autant les laisser à la déchetterie. Ces documents sont mis à disposition, pour les gens. Ils me préviennent à l'avance et s'ils ont besoin de renseignements, s'ils ont un thème particulier j'essaie de leur regrouper le maximum de documents. Les généalogistes, eux, voient l'histoire autrement puisqu'ils font des recherches sur leurs familles, leurs descendants. Moi je complète avec des photos ou des documents.

Il est vrai que si je donne aux Archives une facture comme j'ai là en ce moment sur ma table, "Le Grand Hôtel de Genève", ça n'a peut-être pas un grand intérêt pour l'instant, mais peut-être que dans cinquante ou soixante ans on aura quelqu'un qui fera une étude sur le commerce ou des choses comme ça et sera intéressé par ce document, qui pour le moment est sans intérêt. Il y a eu des courriers qui ont servi pour relater l'histoire locale, la libération de Lons, il y a eu des documents administratifs des Ponts et Chaussées, dont se sert une personne pour avoir son diplôme d'ingénieur. J'ai récupéré les plans du thêâtre de Lons-le-Saunier, alors que personne ne retrouvait les plans originaux après son incendie. Et peut-être d'autres choses qui n'ont pas encore été sorties parce que, avec la quantité..."

\section{"pousser les gens un petit peu»}

Avec sa pratique de l'exposition, M. Rebugai semble étrangement proche de certaines de celles qui ont cours dans l'art contemporain. À partir de certaines affinités décelées avec les questions que posent certains artistes, et sans vouloir hisser $M$. Rebugai au rang d'artiste méconnu, la comparaison permet d'éclairer et de donner une portée à ses pratiques a priori mineures.

Sous le signe de la post-production ${ }^{12}$, Nicolas Bourriaud regroupe ces artistes contemporains qui, depuis le début des années 1990 reproduisent, ré-exposent ou utilisent soit des œuvres réalisées par d'autres, soit des produits culturels disponibles. Toutes ces pratiques, bien que formellement hétérogènes, ont pour point commun de recourir à des formes déjà produites. "La question n'est plus: "que faire de nouveau?" Mais

11. C'est-à-dire un habitant de Lons-le-Saunier.

12. Qui ne désigne ni négation ni dépassement, mais un ensemble d'activités ayant cours, les arts audiovisuels par exemple. 
plutôt: "que faire avec?" Autrement dit, écrit Bourriaud, comment créer du sens à partir de cette masse chaotique d'objets, de noms propres et de références qui constituent notre quotidien? Ainsi, les artistes actuels programment-ils des formes, davantage qu'ils n'en composent ${ }^{13}$."

Non seulement parce que M. Rebugai fait des expositions, mais aussi à la visite de sa cave à papiers, l'endroit où il aime se réfugier, on ne peut s'empêcher de faire le rapprochement avec les installations de Jason Rhoades qu'évoque Nicolas Bourriaud: «l'image affolante d'un monde sans centre possible, qui croule de tous côtés sous le poids de la production et l'impossibilité pratique du recyclage. En le visitant, on pressent que l'art n’a plus pour tâche de proposer une synthèse artificielle des éléments hétérogènes, mais de générer des "masses critiques" formelles à travers lesquelles la structure familiale du marché se métamorphose en un immense entrepôt pour vente en ligne, voire en monstrueuse cité du rebut ${ }^{14}$.»

"Je fais aussi des expositions gratuites. Par contre quand vous faites une exposition, si vous ne mettez que du papier c'est la catastrophe, les gens passent: "il n'y a rien d'intéressant". Vous mettez des photos, c'est pareil ils s'en foutent. Mais si vous mettez un petit décalitre ou un jouet de gosse, ils viennent voir: "Ah oui, tu te rappelles, le grand-père avait le même". Ils commencent à lever la tête, ils lisent la première lettre, ça y est, c'est foutu, ils restent trois heures, ils vous lisent l'expo entière. Si vous ne mettez que de l'écriture: "ça ne nous intéresse pas, c'est complètement débile". Ils passent et ils ne regardent pas. Il faut un élément déclencheur, qui leur rappelle un moment ou qu'ils ont aperçu quelque part. Dès que vous les attirez avec cet élément-là, ça va tout seul. Mais ça c'est pareil on apprend. Ce sont des expositions à thème, l'école ou la dernière guerre par exemple. Pour les cent ans de l'école publique dans un petit pays à la limite de l'Ain, on a ressorti les vieux plumiers, les vieux cartables, les vieux cahiers. Moi, j’ai fourni les documents administratifs. Les gens sont en général bien contents de voir ces expositions. Ils se remettent un petit peu dans l'époque, ça leur rappelle un bon moment. Un moment particulier qui est écrit sur un document administratif et qui déclenche la mémoire: "ah! oui, je me souviens, c'était à telle époque etc." Donc d'un document administratif de deux lignes parfois, qui est sans intérêt, ça remet la mémoire collective en route et on a un fait divers qui n'est pas important mais qui fait vivre cette périodelà. Il est vrai que les grands musées c'est d'autres documents. Ça n’a rien à voir, mais ça permet de pousser les gens un petit peu, vers quelque chose de nouveau, les pousser à aimer un livre, à voir autre chose. Et peut-être après à aller voir les grands musées dont ils ne franchiraient pas la porte parce qu'il y a le côté argent et le côté manque de connaissance. Ce n'est pas la même prétention."

13. Nicolas Bourriaud, Postproduction, Dijon, Les Presses du réel, 2003, p. 9. 14. Ibid., p. 24. 


\section{"...limiter la mémoire à certains individus?»}

Le dispositif créé par M. Rebugai - collecte, tri et stockage - est fortement attaché à la personne de son créateur qui cependant constitue, au-delà de son seul plaisir, son activité comme un geste civique; quil se faufile dans les manquements de la collecte publique ou vise à la constitution appareillée d'une mémoire sans manque, disponible pour tous - qu'ils souhaitent ou non y accéder - et sans zone d'ombre volontairement projetée. Une entreprise singulière qui vise, paradoxalement, à l'intérêt général.

"Moi ce que j'aimerais maintenant, c'est qu'il y ait des gens plus importants que moi qui disent qu'il faut que nous prenions conscience que le papier est un patrimoine, que nous avons des choses intéressantes dessus, et que par exemple sur les chaînes de tri il y ait des gens qui récupèrent ce papier. Parce que je suis sûr que nous laissons partir des documents historiques importants. Moi, $j$ 'ai récupéré des lettres de Georges Trouillot ${ }^{15}$ quand il était ministre des colonies, à l'époque, ces papiers ça partait à la poubelle, maintenant ça finit sur les tapis du tri.

Je ne dirais pas que j’ai une mission. La mission, c'est de vivre une passion. Simplement je pense quiil faut conserver des traces, conserver notre mémoire pour la transmettre à nos enfants, mais une mission, non quand même pas. C'est un loisir qui prend beaucoup de temps, après on verra si on peut exploiter intelligemment ou pas ce loisir. Je pense que les gens préferent ne pas garder de traces du quotidien ou de choses comme ça. Je pense que les gens veulent se laver d'une certaine mémoire, et il est plus facile de refaire des choses quand on ne sait rien, que quand on a des faits. Et je pense que la mémoire c'est aussi quelque chose de gênant, même sur des banalités. Comme je vous l'expliquais sur l'histoire des pièces de dix francs avec l'alliage des deux métaux, je me disais que c'était sympa, qu'ils avaient découvert quelque chose de nouveau, mais en discutant, en me rendant dans des déchetteries, j’ai appris qu’en 1905 ou 1908 il y avait les mêmes pièces. Maintenant si on n'a plus de documents, si l'on n'a plus de traces, on dira qu'on vient de découvrir quelque chose de sensationnel. Il y a plein de choses comme ça, on a toujours l'impression que l'on invente mais on reprend beaucoup ce que nos grands-parents ou arrière-grands-parents avaient commencé à mettre en route ou à fabriquer avec leurs connaissances et leurs moyens.

C'est peut-être une volonté de vouloir faire qu'il n'y ait plus de trace, plus de mémoire. Mais c'est dommage parce qu'ils sont quand même bien contents d'exploiter le savoir de nos grands-parents pour certaines choses. Ce n'est donc pas totalement inutile ou alors est-ce quil faut limiter la mémoire à une certaine quantité d'individus?»

15. Georges Trouillot (1851-1916), élu député du Jura, est connu pour avoir été le rapporteur à la Chambre des députés du projet de loi de 1901 sur les associations. Il fut également sénateur du Jura de 1906 à 1916 et plusieurs fois ministre.

N. Barbe, J.-C. Sevin, «Rencontre avec un braconnier... ", S. \& R., n 19, Avril 2005, pp. 65-75. 
Le texte qui précède vise moins à généraliser qu’à éclairer une singularité et une rencontre. La condition de cette rencontre fut l'hospitalité de M. Rebugai qui l'a acceptée et nous a accueillis dans son territoire et ses cartographies proliférantes.

Ensuite, loin de renvoyer la singularité du côté d'une curiosité que des scientifiques nomades viendraient fixer et épingler en un cabinet pour faire série ou collection, nous nous adressons, chercheurs en sciences sociales, à des êtres «capables de prendre position quant à la pertinence des questions qui leur sont adressées 16 \%. Ce qui nous amène tout à la fois à une conséquence et un constat. La conséquence tout d'abord: au lieu d'un jeu binaire de questions-réponses, c'est la création d'un espace d'énonciation que nous avons tentée, où le problème est négocié en commun, où l'autre fabrique ses questions autant que nous les suscitons et y répond ${ }^{17}$. Le constat ensuite: de la même manière que le musée d'archéologie a traité des modalités d'élection d'objets au rang d'objets muséographiques et de la nature des acteurs qui interviennent dans son espace, M. Rebugai nous parle de ce qui constitue une archive et de ceux qui la définissent. Ce n'est pas un creux que nous lui aurions dessiné qu'il vient remplir. Il affirme là une position et participe au débat quil a contribué à initier.

Nous avons en quelque sorte chassé en bande, pour reprendre les mots de Gilles Deleuze, produisant tout à la fois un objet commun mais ramenant aussi notre propre butin ${ }^{18}$, livré ici, à savoir l'énonciation, par notre braconnier ${ }^{19}$, de ce qui nous/lui semble questionner l'archivation.

16. Isabelle Stengers, Pour en finir avec la tolérance. Cosmopolitiques VII, Paris, La Découverte/Les Empêcheurs de penser en rond, p. 104.

17. «[...] chacun rencontre l'autre, un seul devenir qui n'est pas commun aux deux, puisqu'ils n'ont rien à voir l'un avec l'autre, mais qui est entre les deux, qui a sa propre direction, un bloc de devenir, une évolution a-parallèle» (Gilles Deleuze, Claire Parnet, Dialogues, Paris, Flammarion, 1996, p. 13).

18. Ibid., p. 16.

19. Au fait Monsieur Rebugai, nous vous ferons bien sûr passer un exemplaire de ce texte. Nul besoin cette fois de braconner... Amitiés à vous. 